\title{
Neurodegeneration and neuroprotection in the epileptic brain Chrysanthy Ikonomidou
}

\author{
Address: Department of Pediatric Neurology, University Children's Hospital Carl Gustav Carus University of Technology Dresden Dresden, \\ Germany. \\ from International Society on Brain and Behaviour: 3rd International Congress on Brain and Behaviour \\ Thessaloniki, Greece. 28 November - 2 December 2007 \\ Published: 17 April 2008 \\ Annals of General Psychiatry 2008, 7(SuppI I):S73 doi:10.II86/I744-859X-7-SI-S73
}

This abstract is available from: http://www.annals-general-psychiatry.com/content/7/SI/S73

(C) 2008 Ikonomidou; licensee BioMed Central Ltd.

It was back in the 19th century when pathologists observed hippocampal damage in autopsy studies of patients with epilepsy. In the 1970s it was demonstrated that status epilepticus can cause neuronal degeneration in experimental animal models of recurrent seizures. Currently known mechanisms that underlie epileptic brain injury include acute passive excitotoxic neuronal death as well as active cell death processes triggered by excessive glutamate in the extracellular space, oxidative stress or inflammatory mediators.

In the past decade increasing evidence has accumulated demonstrating that not only status epilepticus but also briefer episodes of recurring seizures may also cause neural degeneration and cognitive dysfunction. With the use of sensitive MR imaging methods, specifically MR volumetry, prospective longitudinal studies in patients are beginning to provide indications that recurring seizure injury in a subset of patients is associated with hippocampal volume loss in the absence of initial events. Seizure suppression and anticonvulsant treatment can prevent adverse structural and functional effects of seizures. Thus, seizure control with anticonvulsants and new medications with neuroprotective properties is desirable.

Recent findings on potentially damaging effects of antiepileptic drugs in the developing mammalian brain need to be taken into consideration when designing antiepileptic therapies for infants, young children and pregnant women. 